\title{
Previsão em Sistemas Dinâmicos Caóticos com Ruído
}

\author{
Wilson Salustiano Júnior ${ }^{1}$, Hugo L. D. de S. Cavalcante ${ }^{1,2}$ \\ ${ }^{1}$ Programa de Pós-Graduação em Modelagem Matemática e Computacional \\ ${ }^{2}$ Departamento de Sistemas de Computação \\ Centro de Informática - Universidade Federal da Paraíba \\ hugo.cavalcante@ci.ufpb.br
}

\begin{abstract}
Resumo: Um modelo muito comum para sistemas dinâmicos é que sua evolução seja dada por uma equação diferencial. Entretanto, a ocorrência de caos determinístico dificulta a aplicação deste modelo para fazer previsões em sistemas reais. Caos determinístico é um fenômeno com dependência sensível a perturbações, o que causa imprevisibilidade a longo prazo. Mostramos como usar dados de séries temporais de um sistema para estimar as equações diferenciais que determinam sua evolução. Usamos como exemplo o sistema de Lorenz e métodos numéricos que incluem o uso de funções polinomiais para ajustar as velocidades das variáveis do estado, e assim determinar um modelo para a sua evolução temporal. Mostramos também que pode ser usado mesmo na situação de dados com ruídos observacionais.
\end{abstract}

Palavras-chave: caos determinístico; regressão linear; previsão em sistemas dinâmicos.

\section{Introdução}

Muitos comportamentos naturais e artificiais podem ser modelados matematicamente como sistemas dinâmicos de equações diferenciais em que determinam a evolução temporal do estado do sistema. No entanto, às vezes é complicado determinar qual o modelo matemático capaz de reproduzir um determinado comportamento físico desejado, especialmente quando tratamos de dados limitados ou de sistemas complexos, onde há a presença da não-linearidade, caos e de ruídos observacionais [1].

Nesse contexto, torna-se interessante desenvolver uma técnica que procure determinar um sistema de equações diferenciais equivalentes ao sistema desconhecido. Uma vez descoberto este sistema existe a possibilidade de se resolver as equações a partir do vetor de estado atual, conhecido, e desta forma fazer estimativas sobre o seu estado futuro [2,3,4].

Técnicas modernas de inteligência artificial, tais como aprendizagem de máquina, amostragem compressiva, e computação em reservatório, têm se revelado eficazes em situações de alta complexidade, resolvendo problemas que constituem desafios para a computação até há pouco. Neste trabalho, usamos algumas dessas técnicas para testar os limites da previsão em um sistema [2,3,5].

Para tentar realizar essas técnicas em dados de sistemas desconhecidos, escolhemos como base um sistema dinâmico simples e caótico, conhecido como sistema de Lorenz. Nossa metodologia consiste em gerar dados observacionais, correspondendo às séries temporais do sistema de Lorenz, usar estes dados para estimar a equação diferencial, bem como os seus parâmetros, que regem a evolução do sistema. Isso é feito com o uso de funções polinomiais, capazes de se ajustar à dinâmica desconhecida. Em seguida, usamos este modelo para prever o estado futuro durante um certo horizonte de previsão. Esta previsão pode ser comparada com o estado verdadeiro do sistema original para testar a precisão da técnica [6].
O objetivo é desenvolver algoritmos capazes de se adaptar ao sistema, com parâmetros flexíveis, que simulem e apresentem soluções aproximadas dos dados coletados.

\section{Metodologia}

Os métodos são implementados em linguagem de programação Python, fazendo uso de módulos préexistentes para análise numérica e ajuste de funções. Usamos as bibliotecas Numpy, Scipy, Random e Matplotlib.

Dado o sistema de Lorenz na equação (1),

$$
\begin{gathered}
\left\{\begin{array}{l}
\dot{x}_{1}=f_{1}, \\
\dot{x}_{2}=f_{2}, \\
\dot{x}_{3}=f_{3},
\end{array}\right. \\
\left\{\begin{array}{l}
f_{1}=\sigma\left(x_{2}-x_{1}\right), \\
f_{2}=r x_{1}-x_{2}-x_{1} x_{3}, \\
f_{3}=x_{1} x_{2}-b x_{3},
\end{array}\right.
\end{gathered}
$$

onde $\sigma=10, r=28$ e $b=2,667$. Encontramos soluções numéricas para este sistema através do método de Runge-Kutta de quarta ordem (RK4) com as condições iniciais adequadas, mas genéricas. A partir desse momento vamos supor que esses dados, considerados como observações exatas, tenham se originado de um sistema dinâmico desconhecido, o qual desejamos estimar e prever.

Temos o objetivo de recuperar a dinâmica do sistema a partir das velocidades desses dados (suas derivadas com respeito ao tempo). A metodologia pode ser dividida em três etapas:

1) Calculamos funções polinomiais unidimensionais de grau $G$ que aplicadas ao tempo se ajustem aos dados observados. Definimos estas como $\boldsymbol{P}(t[n])$, onde 
o tempo foi discretizado. Para encontrar estas funções usamos o método de B-Spline [7].

2) Realizamos uma derivação com relação ao tempo dessas funções polinomiais unidimensionais, chamando a derivada temporal de $\boldsymbol{V}(t[n])$, como mostra na equação (2).

$$
\dot{\boldsymbol{P}}(t[n])=\boldsymbol{V}(t[n]) .
$$

3) Usando o método de Mínimos Quadrados [8], calculamos funções polinomiais multidimensionais $\boldsymbol{F}$, de grau $G$, aplicadas aos dados ajustados, tais que estas funções expressem as velocidades como função das variáveis de estado, como na equação (3).

$$
\boldsymbol{F}(\boldsymbol{P}(t[n]))=\boldsymbol{V}(t[n]) .
$$

Em situações reais, é inevitável que haja erros nos dados, que chamamos de ruído. Para simular a situação de ruído observacional nos dados, acrescentamos a uma cópia dos dados, valores aleatórios com distribuição gaussiana. Isso será feito com a equação (4).

$$
x_{r}=x+r,
$$

onde $\boldsymbol{x}_{r}$ são os dados contaminados por ruído, $\boldsymbol{x}$ são os dados exatos e $\boldsymbol{r}$ são os valores aleatórios produzidos com a distribuição especificada.

\section{Resultados}

Resolvemos a equação (1), com o método RK4, usando as condições iniciais da equação (5).

$$
\left\{\begin{array}{l}
x_{1}[0]=0,1, \\
x_{2}[0]=0,1, \\
x_{3}[0]=0,1,
\end{array}\right.
$$

em um tempo discretizado sobre um passo constante $\Delta t=10^{-2}$. Inicialmente, com $t[0]=0,0$ e $t[N]=100,0$, onde $N$ é o índice final da série. Isso produzirá séries temporais, onde os dados finais estão fora do transiente inicial. Logo em seguida, usamos os dados finais dessas séries como sendo as novas condições iniciais e novamente solucionamos o sistema no mesmo intervalo. Assim obtemos os dados mostrados na Figura 1.

Acrescentamos ruído observacional $r$, com distribuição normal de média $\quad \mu=0 \quad$ e desvio padrão

$\boldsymbol{D}=$ máx $(\boldsymbol{x})$. Só então partimos para a recuperação do sistema usando os dados das séries temporais com ruídos.

Aqui é preciso ter cautela ao se ajustar as funções polinomiais sobre os dados contaminados por ruído. Não queremos que as equações estimadas acompanhem as oscilações rápidas causadas pelo ruído, mas apenas a dinâmica original do sistema, que varia em uma escala de tempo mais lenta. Sendo assim, usamos funções polinomiais unidimensionais $\boldsymbol{P}_{r}(t)$ de grau $G$ que se ajustam com o método B-Spline usando um parâmetro de suavização s. Esse método usa um ajuste de mínimos quadrados de modo que tenta filtrar o ruído, admitindo um resíduo no ajuste de valor $\mathrm{s}$, de acordo com a equação (6).

$$
\sum_{i=1}^{n}\left(\boldsymbol{P}_{\boldsymbol{r}}[n]-\boldsymbol{x}_{\boldsymbol{r}}[n]\right) \leq s,
$$

onde $\boldsymbol{P}_{r}[n]$ é o vetor de funções que desejamos, $\boldsymbol{x}_{\boldsymbol{r}}[n]$ é vetor de dados conhecidos.

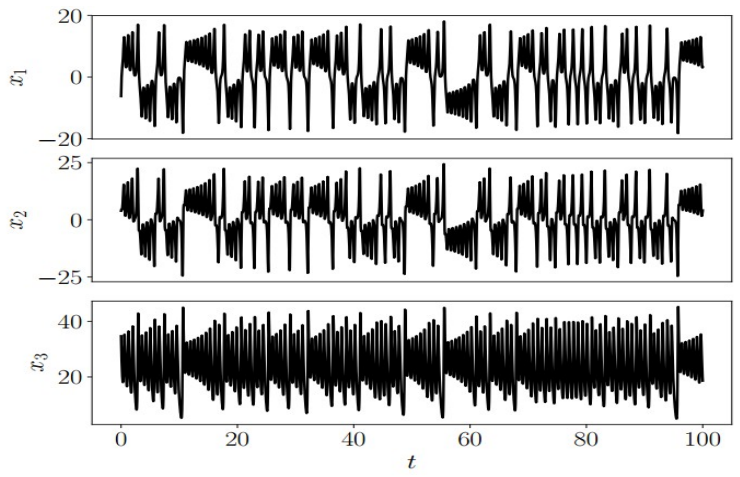

Figura 1. Séries temporais do sistema de Lorenz.

Usando os dados conhecidos, testamos diversos valores de $s$ a fim de chegar ao menor resíduo possível com respeito aos dados exatos, livres de ruído. Sendo assim, acompanhamos a dependência em $s$ do valor do desvio médio ao valor exato $Q$, como na equação (7).

$$
Q=\frac{1}{N} \sum_{i=1}^{n}\left|\boldsymbol{P}_{r}[n]-\boldsymbol{x}[n]\right| \simeq 0
$$

Como o sistema de Lorenz é tridimensional, fazemos

\begin{tabular}{|c|c|c|c|}
\hline \multirow{2}{*}{ 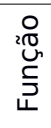 } & \multicolumn{3}{|c|}{ Grau } \\
\hline & 3 & 4 & 5 \\
\hline$P_{1}$ & $s=300$ & $s=300$ & $s=300$ \\
\hline$P_{2}$ & $s=540$ & $s=530$ & $s=530$ \\
\hline$P_{3}$ & $s=1800$ & $s=1800$ & $S=1800$ \\
\hline
\end{tabular}
o uso de três funções polinomiais. O grau $G$ dessas funções polinomiais variando de 3 a 5 . Nossos testes mostraram o melhor valor de $s$ encontrado para cada grau analisado no Quadro 1.

Quadro 1. Análise da escolha do valor de s. FONTE: os autores.

Com o uso desses valores de $s$, seguimos as etapas da metodologia. Na primeira etapa precisamos saber qual o melhor grau para o ajuste dos polinômios e na terceira etapa precisamos saber qual a melhor norma para o ajuste, sempre visando o melhor saldo de previsão. Para definir um critério numérico para a capacidade de previsão arbitramos o tempo (ou horizonte) de previsão como sendo aquele no qual o erro percentual das séries temporais originais e recuperadas diante de ruídos atinge $10 \%$ do valor 
máximo da série. Fizemos uma análise estatística baseada em 10 realizações em que a cada realização mudamos os valores aleatórios de ruídos. Esta análise, mostrando os efeitos do grau e da norma no tempo de previsão, é mostrada na Figura 2.

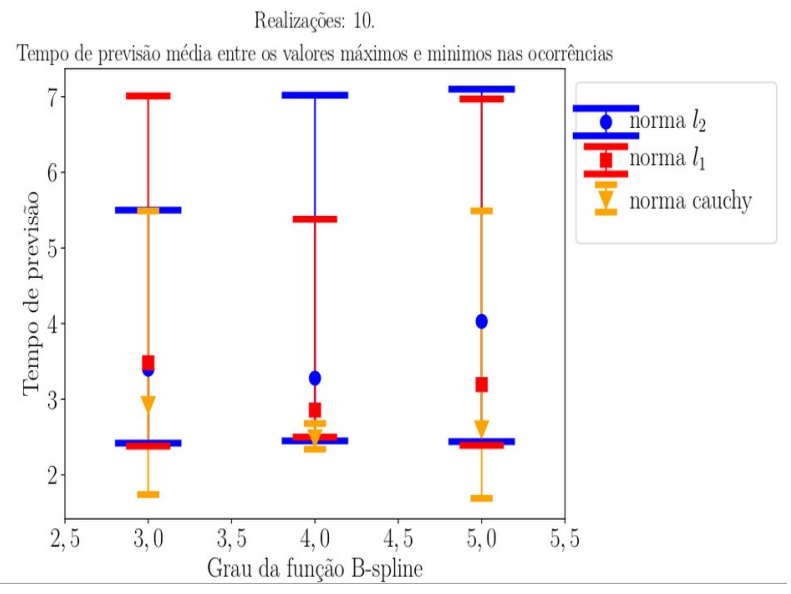

Figura 2. Análise sistemática do grau e norma.

Além disso, sabemos que o tempo de previsão pode ser fortemente influenciado pela condição inicial, pois o tempo de previsão máximo teórico depende do expoente de Lyapunov local observado no espaço de fase, o qual é determinado pelo tempo de observação e pela condição inicial. Fizemos, então, o teste com diferentes condições iniciais, mas fixando os valores de ruído, apresentado na Figura 3.

$10 \mathrm{CI}$

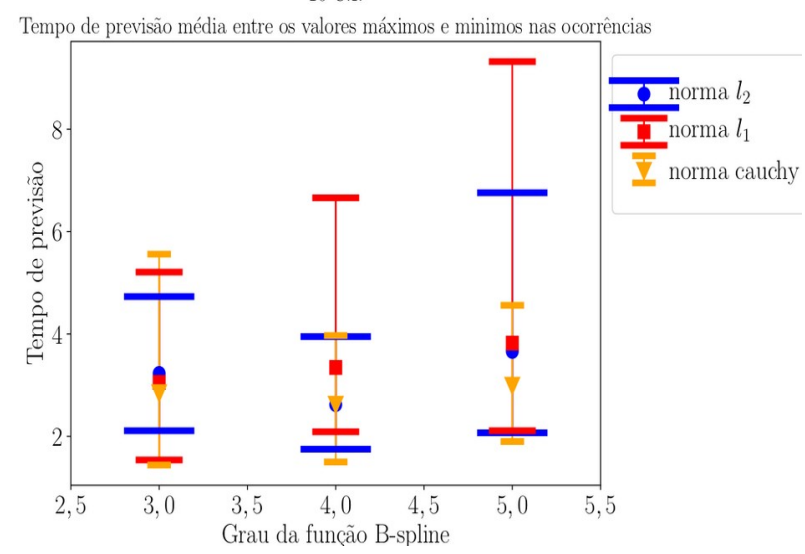

Figura 3. Análise sistemática do grau e norma.

Em seguida, fixando valores de ruídos e com o resultado da análise, fizemos, na primeira etapa, o uso de funções polinomiais unidimensionais de grau 5 . $\mathrm{Na}$ segunda etapa, fazemos uma derivação com relação ao tempo dessas funções e obtemos funções polinomiais unidimensionais de grau 4. Na terceira etapa, fazendo o uso de funções polinomiais multidimensionais de grau 2 e usando a norma $l_{1}$, temos o resultado para os coeficientes dos polinômios, mostrado no Quadro 2.
Sabendo que as funções do sistema de Lorenz são também polinômios, é possível realizar a comparação com os valores exatos. Tratando agora nosso sistema dado por essas novas funções polinomiais, realizamos uma solução numérica através do método RK4.

Quadro 2. Valores resultantes para os coeficientes diante de ruído.

\begin{tabular}{|c|c|c|c|}
\hline \multirow{2}{*}{$\begin{array}{c}\text { 㐫 } \\
\text { ¿ }\end{array}$} & \multicolumn{3}{|c|}{ equação } \\
\cline { 2 - 4 } & $\dot{x}_{1}=f_{1}$ & $\dot{x}_{2}=f_{2}$ & $\dot{x}_{3}=f_{3}$ \\
\hline 1 & -1 & $-0,9$ & $-1,7$ \\
\hline$x_{1}$ & $-10,2$ & 27,8 & $-0,5$ \\
\hline$x_{2}$ & 10 & $-0,8$ & 0,4 \\
\hline$x_{3}$ & $-0,2$ & 0 & $-2,4$ \\
\hline$x_{1} x_{2}$ & 0 & 0 & 1 \\
\hline$x_{1} x_{3}$ & 0 & $-0,9$ & 0 \\
\hline$x_{2} x_{3}$ & 0 & 0 & 0 \\
\hline$x_{1}^{2}$ & 0 & 0 & 0 \\
\hline$x_{2}^{2}$ & 0 & 0 & 0 \\
\hline$x_{3}^{2}$ & 0 & 0 & 0 \\
\hline & & & \\
\hline & 0 & 0 & 0 \\
\hline
\end{tabular}

A Figura 4 apresenta o comparativo das séries do sistema de Lorenz e as séries do sistema aproximado. A Figura 5 apresenta o tempo de previsão de aproximadamente 2,61 unidades de tempo considerando o critério de $10 \%$ do erro percentual das séries temporais originais e recuperadas diante de ruídos.

\section{Discussão}

A análise da Figura 2 e da Figura 3 mostra que para a primeira etapa o melhor grau é 5 e para a terceira etapa a melhor norma é $l_{1}$. Não testamos que efeitos podem ter nesta análise a variação do intervalo de tempo entre os dados temporais e o acréscimo do ruído. O nível de ruído usado foi de apenas $1 \%$ do valor máximo da série temporal Para outros dados, provavelmente os resultados de $s$, graus das funções e normas serão outros. Entretanto, a metodologia é a mesma, o que nos assegura sua eficácia. Também em situações reais, onde não conhecemos o sistema, a tarefa de encontrar o valor de suavização torna-se mais complicada, pois não podemos calcular $Q$, o desvio do valor exato, mas o uso de B-Spline ajustada possibilita estimar um valor de $s$ adequado de modo a não ajustar o ruído dos dados. 


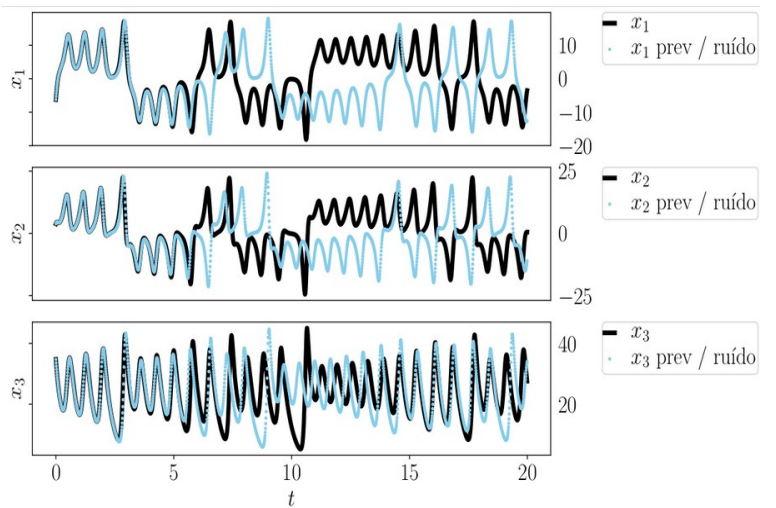

Figura 4. Comparação entre as séries temporais originais e as séries recuperadas diante de ruído.

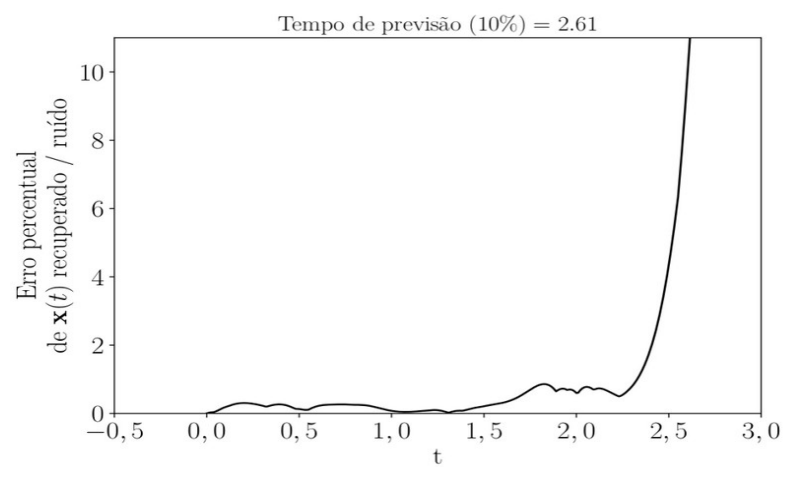

Figura 5. Erro percentual das séries temporais originais e recuperadas diante de ruídos. FONTE: os autores.

\section{Conclusões}

Usamos o sistema de Lorenz como exemplo para aplicar e desenvolver o nosso método de previsão em sistemas dinâmicos. Mostramos que o conhecimento de séries temporais de dados oriundos do sistema pode ser usado para recuperar a dinâmica que origina o comportamento observado. Em seguida, usando a dinâmica recuperada, e abordamos o problema da previsão do estado futuro.

Desenvolvemos uma técnica de inteligência artificial utilizando funções polinomiais para calcular as velocidades de evolução das variáveis de estado do sistema e com isso, recuperamos suas equações diferenciais e finalmente realizamos a previsão. O algoritmo proposto é capaz de se adaptar ao sistema, com parâmetros flexíveis, extraídos a partir dos dados coletados em observações. Sendo assim, os resultados são eficazes diante de um sistema caótico e o método ainda pode ser ajustado e aplicado a outros sistemas. Exploramos algumas situações que se espera encontrar em problemas reais, no intuito de mostrar que sua aplicabilidade prática é viável.

Para reduzir o efeito do ruído, fizemos testes com diferentes valores de um parâmetro de suavização e conseguimos encontrar valores que fazem uma filtragem nos dados com ruídos de modo a aproximá-los cada vez mais dos dados considerados exatos.

Em geral, conclui-se que as aproximações por funções polinomiais são eficazes para recuperar as equações governantes dos sistemas. Logo, conforme temos os métodos para a previsão de um sistema caótico, então é possível sua aplicação a dados reais de sistemas desconhecidos, desde que tenham características similares, tais como determinismo e baixa dimensionalidade.

\section{Bibliografia}

[1] Strogatz, S. (1994) Nonlinear Dynamics and Chaos: With Applications to Physics, Biology, Chemistry, and Engineering. Studies in Nonlinearity. Perseus Books.

[2] Ang, R.; Lai, Y. C.; Grebogi, C. (2012) Forecasting the future: Is it possible for adiabatically time-varying nonlinear dynamical systems? Chaos: An Interdisciplinary Journal of Nonlinear Science 22(3): 033119. DOI $10.1063 / 1.4740057$

[3] Lu, Z.; Hunt, B. R.; Ott, E. (2018) Attractor reconstruction by machine learning. Chaos: An Interdisciplinary Journal of Nonlinear Science 28(6): 061104. DOI: $10.1063 / 1.5039508$

[4] De S. Cavalcante, H. L. D., Oriá, M., Sornette (2013) Predictability and Suppression of Extreme Events in a Chaotic System. Physics Review Letters 111(11): 198701. DOI: $10.1103 /$ PhysRevLett.111.198701

[5] Tran, G.; Ward, R. (2016) Exact Recovery of Chaotic Systems from Highly Corrupted Data. Cornell University. https://arxiv. org/abs/1607.01067>. Acesso em 2019/06/3.

[6] Kantz, H.; Schreiber, T. (2004) Nonlinear Time Series Analysis. Cambridge nonlinear science series. Cambridge University Press.

[7] Pereira, L. R., 2014, Ajuste de curva B-spline fechada com peso. Dissertação de Mestrado, Universidade Federal de Uberlândia, Uberlândia.

[8] Ruggiero, M.; Rocha Lopes, V. (1996) Cálculo numérico: aspectos teóricos e computacionais. Pearson Makron Books. 